\title{
Efecto del programa Fifa $11+$ en la flexibilidad de los cadetes de nivel v de la Facultad de Educación Física Militar de la Escuela Militar de Cadetes General José María Córdova*
}

\author{
Brayan Camacho Arcila** \\ Iván Colmenares Murcia** \\ Carlos Reyes Forero*** \\ Omar Danilo Guerrero Osma****
}

Recibido: abril 19 del 2019 • Aceptado: agosto 21 de 2019

\begin{abstract}
"Artículo de investigación, desarrollado en el macroproyecto de investigación Efectos del programa Fifa 11+ sobre las capacidades físicas del cadete de la Escuela Militar, desarrollado en la Escuela Militar de Cadetes General José María Córdova, con recursos financieros propios de los investigadores. Citar como: Camacho, A., Colmenares, I., Reyes, C. y Guerrero, O. (2019). Efecto del programa Fifa 11+ en la flexibilidad de los cadetes de nivel v de la Facultad de Educación Física Militar de la Escuela Militar de Cadetes General José María Córdova. Revista de Investigación Cuerpo, Cultura y Movimiento, 9(2), 21-35. DoI: https:// doi.org/10.15332/2422474x/5357
\end{abstract}

"* Estudiante de Ciencias Militares y Educación Física Militar en la Escuela Militar de Cadetes General José María Córdova, Colombia. Correo electrónico: brayancam@outlook.es / ORCID: 0000-0002-6208-2094

"** Estudiante de Ciencias Militares y Educación Física Militar en la Escuela Militar de Cadetes General José María Córdova, Colombia. Correo electrónico: colmenaresivandavid@ hotmail.es / ORCID: 0000-0002-4616-7215

"**** Estudiante de Ciencias Militares y Educación Física Militar en la Escuela Militar de Cadetes General José María Córdova, Colombia. Correo electrónico: carlosreyes9524@gmail. com / ORCID: 0000-0002-5858-1237

**:* Magíster en Fisioterapia del Deporte y la Actividad Física, docente de la Facultad de Educación Física Militar, tutor e investigador principal del macroproyecto, Colombia. Correo electrónico: omardguerrero@gmail.com / ORCID: 0000-0001-9095-2651 


\section{Resumen}

El objetivo de este estudio fue analizar el efecto del programa de calentamiento Fifa 11+ en los cadetes de nivel V de la Facultad de Educación Física Militar (FEFM) de la Escuela Militar de Cadetes (Esmic) y su relación con la flexibilidad. El estudio fue experimental, descriptivo y tipo ensayo clínico. Se realizó la evaluación por medio del test sit-and-reach con cajón; luego, se implementó el programa de calentamiento Fifa 11+ al grupo experimental 3 veces por semana, durante 10 semanas. Los datos estadísticos fueron analizados por medio del modelo ANOva factorial. Los resultados indicaron que el grupo experimental mejoró la flexibilidad en $67.9 \%$, en comparación con $8.2 \%$ del grupo control. Por lo tanto, se llegó a la conclusión de que el programa de calentamiento Fifa 11+ es efectivo para mejorar la flexibilidad en los cadetes de nivel v de la FeFM de la Esmic.

Palabras clave: cadete, Fifa 11+, flexibilidad, personal militar. 


\section{Effect of Fifa $11+$ program on the flexibility of level $v$ cadets of the Faculty of Military Physical Education of the General José María Córdova Military Cadet School}

\section{Abstract}

The objective of this research was to analyze the Fifa 11+ warm-up program on level $\mathrm{v}$ cadets of the military school and its relationship with flexibility through a descriptive, experimental and clinical trial study. The evaluation was made by conducting the Sit and Reach test with a drawer; the Fifa 11+ warm-up program was then implemented to the experimental group during 10 weeks, 3 times a week. The statistical data were analyzed by using the ANOVA Factorial model. The results indicate that in the experimental group flexibility improved $67.9 \%$ compared to $8.2 \%$ in the control group. Therefore it was concluded that the Fifa 11+ warm-up program is effective for improving flexibility in level v cadets of the Faculty of Military Physical Education of the Esmic.

Keywords: cadet, Fifa 11+, flexibility, military personnel. 


\section{Introducción}

Según Ríos-Pinillos, Castro-Jiménez y Melo-Buitrago (2016), las escuelas militares en el mundo están encargadas de formar a los futuros oficiales del ejército de un país. Dentro de la formación que los cadetes reciben se encuentra, por un lado, la académica, que busca brindar herramientas para que estos se desarrollen como profesionales en un área determinada dentro del espacio militar; y, por otro, el entrenamiento físico, encargado de desarrollar el cuerpo de los cadetes para poder afrontar diferentes situaciones. Desde lo académico, los cadetes deben entregar trabajos, proyectos, realizar consultas e investigaciones; en la instrucción militar deben completar los entrenamientos, pruebas físicas y demostrar que son aptos para diferentes actividades en la escuela (Rodríguez, Valenzuela, Velasco, Castro y Melo, 2016).

El cadete no viene con una huella de entrenamiento físico - teniendo en cuenta las evaluaciones de ingreso a la escuela-, lo cual implica una forma física inadecuada, que genera que exista en el estudiante una oscilación en cualquiera de sus capacidades físicas. Esto puede conllevar a fatiga, reducción del tiempo de reacción, incoordinación e, incluso, a lesiones osteomusculares que resultan en la incapacidad para realizar entrenamiento físico (Guerrero, 2016).

Un ejemplo de lo enunciado por Guerrero (2016) se ve reflejado en el caso de los jugadores de fútbol, donde las estadísticas han determinado que es el deporte que presenta más lesiones (Raya, 2017), con tasas de 35.9/1000 horas competencia y 9.6/1000 horas entrenamiento (Hootman, Dick y Agel, 2007). Todo esto se evidencia por ser el deporte más practicado y de mayor seguimiento, lo cual lo convierte en una práctica con mayor presión para el deportista que lo práctica. La solución acoplada del programa Fifa 11+ ha sido una alternativa viable en el tema de prevención de estas lesiones musculares y mejoramiento de capacidades físicas.

El programa de calentamiento Fifa 11+ fue desarrollado por el Centro Médico de Evaluación e Investigación de la Fifa (F-MARC, por sus siglas en inglés), a través de ejercicios dinámicos y específicos (Costa, Fernandes, Salvador y Rocha 2015), compuesto por entrenamiento pliométrico, balance y agilidad (Nakase et al., 2013). Este material se encuentra disponible en inglés, alemán, francés, italiano y español (Junge et al., 2010). 
El fútbol es un deporte que cuenta con maniobras de impacto de alta intensidad e intermitentes (Bengoa, López, Prada, González y Yáñez, 2012), así como la cotidianidad del militar, lo que conlleva a la predisposición de lesiones, principalmente osteomusculares. Estas últimas son un problema frecuente dentro de las escuelas militares de Colombia; las de mayor incidencia son: periostitis, tendinitis, fracturas y esguinces (Rodríguez et al., 2016). Los arduos entrenamientos a los que se enfrentan los cadetes, a veces sin previo calentamiento, los hacen proclives a lesiones que afectan su desempeño dentro de la escuela y pueden tener repercusiones a mediano y largo plazo.

La flexibilidad -definida como la capacidad física de amplitud de movimientos de una sola articulación o de una serie de articulaciones (Arregui y Martínez, 2001) - y los movimientos -clasificados atendiendo varios puntos de vista-, así como las manifestaciones de la flexibilidad como expresión de las capacidades humanas de movimiento, pueden ser organizados desde diferentes enfoques (Merino y Fernández, 2009). De igual modo, la valoración de la flexibilidad es una práctica común en el ámbito de la salud físico-deportiva y su reducción ha sido relacionada con alteraciones musculoesqueléticas que generan lesiones en estas estructuras (Ayala, Sainz, Cejudo y Santoja, 2012).

Entendiendo que las lesiones osteomusculares ocurren por la falta de preparación en las capacidades físicas, es necesario encontrar un programa validado, confiable y que no requiera materiales especiales para su ejecución; además, debe ser de fácil aplicación en los cadetes de la Escuela Militar General José María Córdova, para poder analizar sus efectos y así mejorar el rendimiento físico militar. Por esta razón, surge la pregunta: ¿cuál es el efecto del programa Fifa 11+ en la flexibilidad de los cadetes de nivel v de la Facultad de Educación Física Militar (Fefm) de la Escuela Militar General José María Córdova (Esmic)?

El mayor nivel de exigencia en los cadetes se encuentra en la parte física, debido a la importancia que representa para la milicia. Es por esto por lo que los entrenamientos físicos militares están conformados por una serie de ejercicios que ponen a prueba las condiciones del estudiante, llevándolos al límite para poder obtener el máximo potencial. Es así como, a causa del gran nivel de exigencia, los cadetes en formación a lo largo de la carrera experimentan diversas lesiones musculares causadas por falta de calentamiento o malas prácticas en las diferentes capacidades físicas. 
El Fifa $11+$ es un programa de calentamiento que tiene por objetivo prevenir lesiones y mejorar las capacidades físicas del individuo, con una duración de 15 a 20 minutos, y se puede utilizar en entrenamiento y en partidos (Canosa, 2016). Los estudios realizados por Bizzini, Junge y Dvorak (2011), Beijsterveldt, Krist, Schmikli, Stubbe y Inklaar (2011) y SilversGranelli y colaboradores (2015) demostraron la relevancia del programa Fifa 11+ para el mejoramiento de las cualidades físicas en los futbolistas. De acuerdo con lo anterior, el presente trabajo busca realizar un análisis del efecto del programa Fifa 11+ en la flexibilidad de los cadetes nivel v de la FEFM de la Esmic.

\section{Metodología}

La presente investigación se planteó como un estudio experimental descriptivo, tipo ensayo clínico aleatorizado (Ruiz y Morillo, 2004). Se evaluaron dos grupos de cadetes: uno experimental con la aplicación del calentamiento Fifa $11+$ (Dvorak y Junge, 2005) y otro grupo que siguió haciendo su calentamiento cotidiano; los participantes realizaron el calentamiento 3 veces por semana, durante 10 semanas. La investigación se realizó en la Esmic, con cadetes de la Facultad de Educación Física Militar. Los sujetos se seleccionaron a conveniencia, según el objeto de estudio. La población de estudio la constituyó un grupo de 43 estudiantes de nivel v, 21 en el grupo control y 22 en el experimental. Con la aplicación de los criterios de exclusión, se modificó a 37, 17 y 20, respectivamente.

\section{Criterios de inclusión}

- Firmar del consentimiento informado

- Estar inscritos en la Esmic

- Pertenecer al nivel v de la Facultad de Educación Física Militar

- Entrenar 3 o más veces por semana

- No ser de la compañía de deportistas. 


\section{Criterios de exclusión}

- Tener una lesión aguda de los miembros inferiores

- No asistir a las evaluaciones

- Entrenar menos de 3 veces a la semana.

La evaluación de la flexibilidad se realizó por medio del test sit-and-reach con cajón, y se implementó el programa de calentamiento Fifa 11+ al grupo experimental 3 veces por semana, durante 10 semanas. Durante este periodo, el grupo control continuó con su calentamiento habitual. Una vez terminado el tiempo de intervención, se realizó de nuevo la evaluación por medio del test sit-and-reach con cajón, el cual tiene una fiabilidad de 0.890.99 (Ayala, Sainz, Croix de Ste y Santoja, 2012).

La sistematización y análisis de los datos se realizó utilizando el modelo de ANOva factorial de medidas repetidas, el cual, a pesar de no soportar una prueba de normalidad, se puede aplicar para análisis cuantitativos de las evaluaciones pre y posintervención. Se estableció un valor $\mathrm{p} \leq 0.05$ para el análisis de las diferencias significativas entre variables. El estudio se realizó de acuerdo con la Declaración de Helsinki y la Resolución 008430 de 1993 del Ministerio de Salud Protección Social de Colombia, categorizado en riesgo mínimo y respetando la confidencialidad de los datos de los participantes.

\section{Resultados}

En la figura 1 se pueden observar las evaluaciones pre y posintervención del programa de calentamiento Fifa 11 + realizadas a los cadetes de nivel v pertenecientes al grupo experimental. En tono claro se muestran las primeras evaluaciones a los 20 cadetes; y en tono oscuro las segundas, realizadas 10 semanas después. En la primera evaluación se puede evidenciar que los datos de flexibilidad en la muestra son dispersos, pero para la segunda evaluación, posintervención, se encuentra una población más homogénea, además del mejoramiento de la capacidad muscular de flexibilidad. 
Figura 1. Flexibilidad del grupo experimental

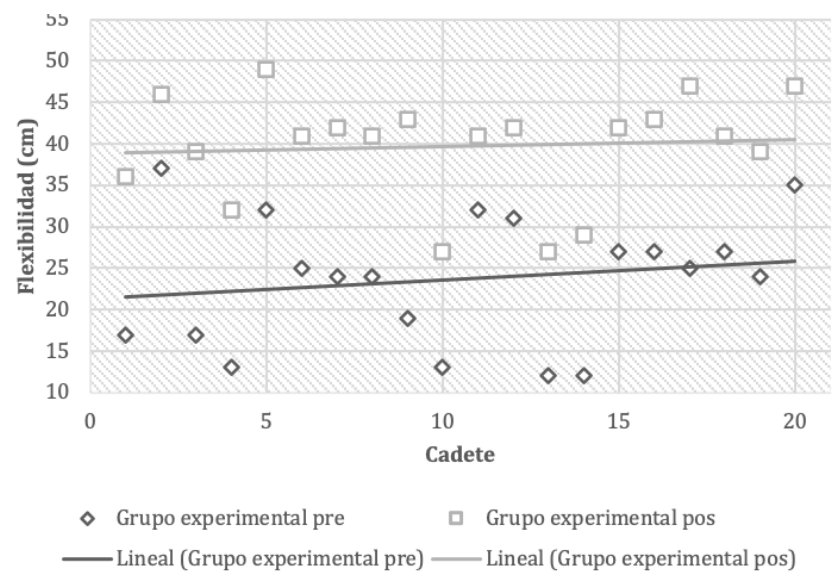

Fuente: elaboración propia.

En la figura 2 se muestra las evaluaciones pre y pos de los cadetes de nivel $\mathrm{v}$ pertenecientes al grupo control. En tono claro se muestra la primera evaluación; y en tono oscuro, la segunda, que se llevó a cabo 10 semanas después de realizar su trabajo habitual dirigido u ordenado por las directrices de la Escuela. En ambas evaluaciones se observa una dispersión de datos de manera irregular.

Figura 2. Flexibilidad del grupo control

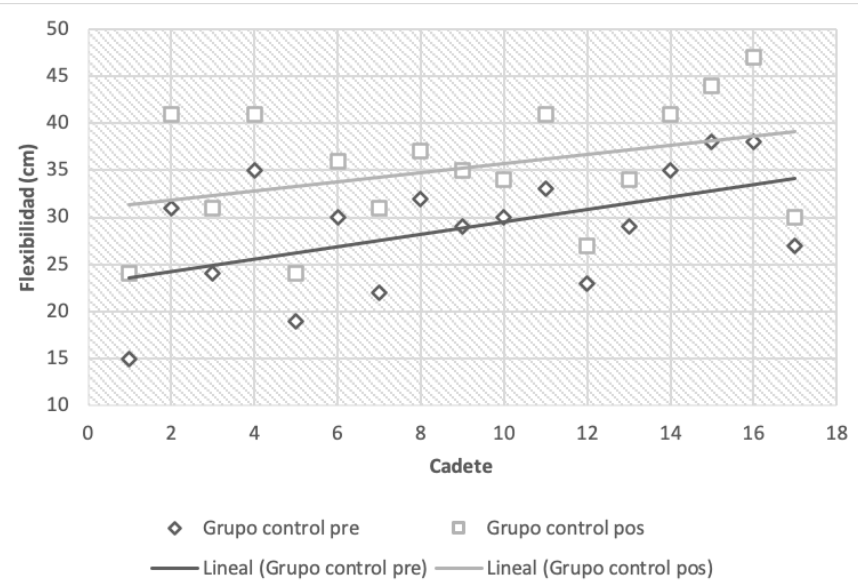

Fuente: elaboración propia. 
En la tabla 1 se presentan los resultados de las evaluaciones en los grupos experimental y control, además del total de población evaluada. Se evidencia que en el grupo experimental hubo una mejoría de 16 centímetros, en comparación con la evaluación preintervención del programa de calentamiento Fifa 11+. Esto representa un $67 \%$, en comparación con el grupo control que, en 10 semanas de calentamiento habitual, mejoró 6 centíme$\operatorname{tros}(22 \%)$.

Tabla 1. Resultados de las evaluaciones pre y posintervención

\begin{tabular}{cccccccc}
\hline \multicolumn{7}{c}{ Flexibilidad } \\
\hline Grupo & N & Pre & DS & Pos & DS & Absoluto & $\begin{array}{c}\text { Cambio } \\
(\%)\end{array}$ \\
Experimental & 20 & 23.65 & 7.77 & 39.70 & 6.44 & 16.05 & 67.8 \\
Control & 17 & 28.62 & 6.49 & 35.17 & 6.79 & 6.55 & 22.9 \\
Total & 37 & 26.02 & 7.57 & 37.62 & 6.90 & 11.06 & 44.6 \\
\hline
\end{tabular}

Fuente: elaboración propia.

En la figura 3 se muestra el grupo experimental en tono oscuro y el grupo control en tono claro. Se puede apreciar la interacción positiva y entender que aplicar el programa Fifa 11+ 3 veces por semana, durante 10 semanas, tiene una mayor probabilidad de mejorar la flexibilidad muscular, en comparación con la población que continúa realizando el calentamiento convencional, que consta de movilidad articular o estiramientos sin un rigor metodológico.

El principal hallazgo de este estudio fue que en 10 semanas de intervención con el programa Fifa 11+ realizado 3 veces por semana, se mejoró significativamente la variable de flexibilidad en el grupo experimental en comparación con el grupo control que mantuvo su rutina normal de entrenamiento (GE+: $67.8 \%$ frente a GC: $22.9 \%$ ). 
Figura 3. Medias marginales estimadas de flexibilidad

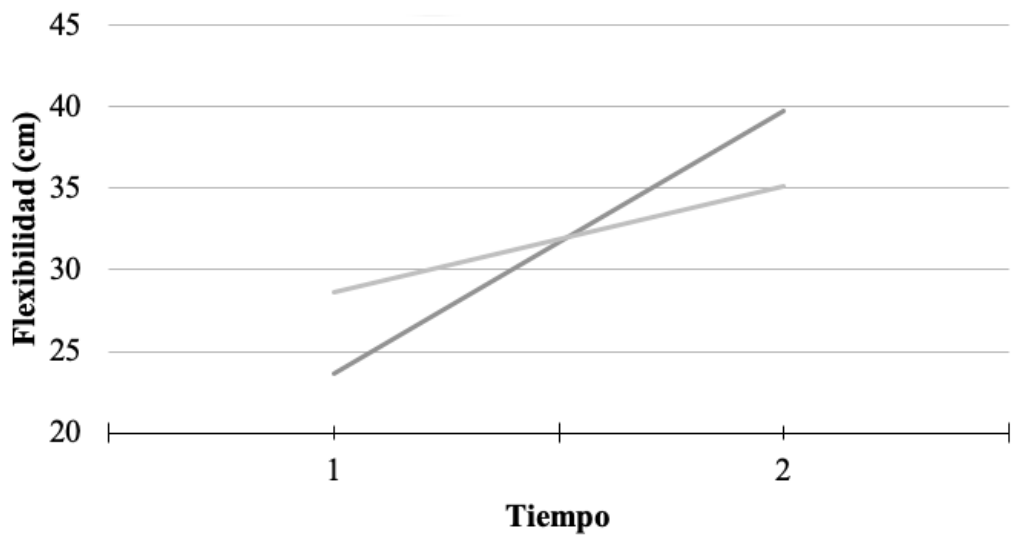

Experimental $\longrightarrow$ Control

Fuente: elaboración propia.

La propuesta de incorporar el programa de calentamiento Fifa 11+ en la rutina de entrenamiento ha dado buenos resultados, en términos de prevención de lesiones (Impellizzeri et al., 2013; Soligard et al., 2008; Fernandes, da Silva, da Costa y Marins, 2015; Barengo et al., 2014), y mejora de los indicadores de desempeño (Daneshjoo, Mokhtar, Rahnama y Yusof, 2013; Brito et al., 2010). Sin embargo, algunos autores sugieren que los estímulos no son suficientes para inducir efectos significativos (Kilding, Tunstall y Kuzmic, 2008; Steffen, Bakka, Myklebust y Bahr, 2008).

Los resultados confirman la hipótesis inicial de que el grupo experimental exhibiría mejor rendimiento de flexibilidad que el grupo control. Estos hallazgos sugieren que la inclusión de Fifa 11+ en la rutina de entrenamiento podría mejorar la capacidad de potenciar el control neuromuscular (Bizzini et al., 2013), que es la interacción de sistemas que integran las diferentes acciones del músculo (estática, dinámica y reactiva), activación muscular (excéntrica y concéntrica), coordinación inter e intramuscular, estabilización del núcleo, equilibrio y postura corporal. 
Además, Ayala, Sainz y Cejudo (2012) concluyen que, en cuanto eficacia se refiere, no parece existir una técnica mejor que otra, por lo que todas (estática-activa, dinámica, de facilitación neuromuscular propioceptiva) parecen ser técnicas eficaces para la mejora del rango de movimiento articular. Por lo tanto, pueden ser utilizadas y combinadas como parte del entrenamiento de la flexibilidad.

El calentamiento Fifa $11+$ se puede considerar un programa de calentamiento adecuado para cadetes de la Esmic, porque es capaz de inducir respuestas fisiológicas positivas y es potencialmente eficaz para reducir los factores de riesgo de lesiones. Además, el Fifa 11+ es más efectivo en comparación con las rutinas de calentamiento convencionales o no estructuradas, como las realizadas en la Esmic, en las que el cadete realiza su propio calentamiento sin una explicación previa de cómo realizarlo.

En el presente estudio la flexibilidad muscular de la zona corporal posterior mejoró significativamente (G11+67.8\%), lo que sugiere un gran efecto del entrenamiento en esta variable. Una posible explicación para la superioridad del grupo experimental en términos de elasticidad está relacionada con el entrenamiento mejorado de las contracciones del grupo muscular core e isquiotibiales, y sus progresiones de carga (Bizzini et al., 2013), que pueden tener mayor producción de fuerza, tal como sucedió en el trabajo realizado por Alves, Borba-Pinheiro, Alencar, Chulvi-Medrano y Dantas (2012). Los resultados de su estudio de entrenamiento de fuerza con judocas, durante 7 semanas, mostraron una mejoría de la flexibilidad del $25.2 \%$. Se encontró entonces que el programa Fifa 11+ es eficaz para promover un aumento considerable en el rendimiento de la flexibilidad. Estos resultados son interesantes para entrenadores y cadetes de la Esmic, ya que este programa de calentamiento puede ser desarrollado sin tiempo y gasto financiero excesivos.

\section{Conclusión}

Se puede concluir que 10 semanas de intervención con el programa de calentamiento Fifa 11+ promovió un aumento significativo en los parámetros neuromusculares (elasticidad) de los cadetes de la FEFM de la Esmic, como lo demuestra la mejora en el performance de flexibilidad en el grupo de 
intervención o experimental. Se recomienda la implementación de este tipo de calentamiento en los diferentes niveles y facultades de la Esmic.

\section{Referencias}

Alves, K., Borba-Pinheiro, C., Alencar, de G., Chulvi-Medrano, I. y Dantas, M. (2012). Fuerza muscular y flexibilidad de judocas masculinos de alto rendimiento que participaron en la liga española durante 2011. Motricidad Humana, 13(1), 28-35.

Arregui, J. y Martínez, V. (2001). Estado actual de las investigaciones sobre la flexibilidad en la adolescencia. Revista Internacional de Medicina y Ciencias de la Actividad Física y el Deporte, 1(2), 127-35.

Ayala, F., Sainz, P. y Cejudo, A. (2012). El entrenamiento de la flexibilidad: técnicas de estiramiento. Revista Andaluza de Medicina del Deporte, 5(3), 105-12.

Ayala, F., Sainz, P., Cejudo, A. y Santoja, F. (2012) Pruebas angulares de estimación de la flexibilidad isquiosural: análisis de la fiabilidad y validez. Revista Andaluza Medicina del Deporte, 5(2), 67-74. DoI: https://doi.org/10.1016/ s1888-7546(12)70011-4

Ayala, F., Sainz de Baranda, P., de Ste Croix, M., y Santonja, F. (2012). Fiabilidad y validez de las pruebas sit-and-reach, revisión sistemática. Revista Andaluza de Medicina del Deporte, 5(2), 57-66.

Barengo, N., Meneses-Echávez, J., Ramirez-Velez, R., Cohen, D., Tovar, G. y Correa, J. (2014). The impact of the FIFA 11+ training program on injury prevention in football players: A systematic review. International Journal of Environmental Research and Public Health, 11(11), 11986-12000. DoI: https://doi.org/10.3390/ijerph111111986

Beijsterveldt, A. M. C. van, Port, I. G. L. van de, Krist, M. R., Schmikli, S. L., Stubbe, J. H., Frederiks, J. E. y Backx, F. J. G. (2012). Effectiveness of an injury prevention programme for adult male amateur soccer players: A cluster-randomised controlled trial. British Journal of Sports Medicine, 46(16), 1114-8. DoI: https://doi.org/10.1136/bjsports-2012-091277

Bengoa, F., López, F., Prada, C., González, R. y Yáñez, F. (2012). Flexibilidad muscular y lesiones musculotendineas en el fútbol formativo masculino de un club deportivo chileno. Archivo Sociedad Chilena de Medicina del Deporte, 57, 7-13. 
Bizzini, M., Impellizzeri, F., Dvorak, J., Bortolan, L., Modena, R. y Junge, A. (2013). Physiological and performance responses to the "FIFA 11+(part 1): is it an appropriate warm-up?” Journal Sports Science, 31(13), 1481-90. DoI: https:// doi.org/10.1080/02640414.2013.802922

Bizzini, M., Junge, A. y Dvorak, J. (2011). Implementation of the FIFA 11+ football warm up program: How to approach and convince the football associations to invest in prevention. British Journal of Sports Medicine, 47(12), 803-806. DOI: http://dx.doi.org/10.1136/bjsports-2012-092124

Brito, J., Figueiredo, P., Fernandes, L., Seabra, A., Soares, J., Krustrup P. y Revelo A. (2010). Isokinetic strength effects of FIFA's "the 11+" injury prevention training programme. Isokinetics and Exercise Science, 18(1), 211-5. DoI: https:// doi.org/10.3233/IES-2010-0386

Canosa Pena, A. (2016). Eficacia del programa FIFA 11+ en la prevención de lesiones deportivas. Recuperado de https://ruc.udc.es/dspace/handle/2183/18505

Costa, J., Fernandes, J., Salvador, P. y Rocha, C. (2015). The effect of "FIFA11+" on vertical jump performance in soccer players. Revista Brasileña Cineantropometria Desempehno Humano, 17(6), 733-41. DoI: http://dx.doi. org/10.5007/1980-0037.2015v17n6p733

Daneshjoo, A., Mokhtar, A. H., Rahnama, N. y Yusof, A. (2012). The Effects of Injury Preventive Warm-Up Programs on Knee Strength Ratio in Young Male Professional Soccer Players. PLoS ONE, 7(12), e50979. Dor: https://doi. org/10.1371/journal.pone.0050979

Dvorak, J. y Junge A. (2005). Manual de Medicina del Fútbol del F-MARC:81-93. Zúrich: Fédération Internationale de Football Association (FIFA).

Fernandes, A., da Silva, C., da Costa, I. y Marins J. (2015). The "FIFA 11+" warp-up programme for preventing injuries in soccer players: a systematic review. Fisioterapia em Movimento, 28(2), 397-405. DoI: https://doi. org/10.1590/0103-5150.028.002.AR01

Guerrero, O. (2016). Efecto del programa FIFA 11+ para futbolistas en la coordinación, balance y fuerza de miembros inferiores (tesis inédita de maestría). Universidad Nacional de Colombia, Bogotá, Colombia. DoI: https://doi. org/10.1016/j.ft.2019.02.003

Hootman, J., Dick, R. y Agel, J. (2007). Epidemiology of collegiate injuries for 15 sports: summary and recommendations for injury prevention initiatives. Journal of athletic training, 42(2), 311-9. Recuperado de https://pubmed.ncbi. nlm.nih.gov/17710181/ 
Impellizzeri, F., Bizzini, M., Dvorak, J., Barbara P., Schena, F. y Junge, A. (2013). Physiological and performance responses to the FIFA 11+ (part 2): a randomized controlled trial on the training effects. Journal Sports Science, 31(13), 1491-502. DoI: https://doi.org/10.1080/02640414.2013.802926

Junge, A., Lamprecht, M., Stamm, H., Hasler, H., Bizzini, M., Tschopp, M.,... Dvorak, J. (2011). Countrywide campaign to prevent soccer injuries in Swiss amateur players. The American Journal of Sports Medicine, 39(1), 57-63. DoI: https://doi.org/10.1177/0363546510377424

Kilding, A. E., Tunstall, H. y Kuzmic, D. (2008). Suitability of FIFA's «The 11» Training Programme for Young Football Players-Impact on Physical Performance. Journal of Sports Science \& Medicine, 7(3), 320-6.

Merino, R. y Fernández E. (2009). Revisión sobre tipos y clasificaciones de la flexibilidad. Una nueva propuesta de clasificación. Revista Internacional de Ciencias de Deporte, 5(5), 52-70. DoI: 10.5232/ricyde2009.01604

Nakase, J., Inaki, A., Mochizuki, T., Toratani, T., Kosaka, M., Ohashi, Y., ... Tsuchiya, H. (2013). Whole body muscle activity during the FIFA 11+ program evaluated by positron emission tomography. PloS One, 8(9), e73898. Dor: https://doi.org/10.1371/journal.pone.0073898

Raya-González, J. (2017). El entrenamiento de fuerza para la prevención de lesiones en el fútbol: revisión sistemática. Revista digital de Educación Física, 9(49), 2335. Recuperado de https://dialnet.unirioja.es/servlet/articulo?Codigo=6195134

Ríos-Pinillos, C., Castro-Jiménez, L. y Melo-Buitrago, P. (2016). Lesiones derivadas del entrenamiento militar en los cadetes de $6^{\circ}$ nivel de la Escuela Militar José María Córdova. Revista Movimiento Científico, 10(1), 19-28. Dor: https://doi. org/10.33881/2011-7191.mct.10102

Rodríguez, J., Valenzuela, J., Velasco, J., Castro, L. y Melo, P. (2016). Caracterización lesiones derivadas del entrenamiento militar. Revista Cuidarte, 7(1), 1219-26. DOI: http://dx.doi.org/10.15649/cuidarte.v7i1.301

Ruiz, A. y Morillo, L. (2004). Epidemiología clínica. Investigación clínica aplicada. Bogotá: Editorial Médica Panamericana.

Soligard, T., Myklebust, G., Steffen, K., Holme, I., Silvers, H., Bizzini, M., Junge, A., Dvorak, J., Bahr, R. y Andersen, TE. (2008). Comprehensive warm-up programme to prevent injuries in young female footballers: cluster randomized controlled trial. British Medical Journal, 337, 1-9. DoI: https://doi. org/10.1136/bmj.a2469

Silvers-Granelli, H., Mandelbaum, B., Adeniji, O., Insler, S., Bizzini, M., Pohlig, R., ... Dvorak, J. (2015). Efficacy of the FIFA 11+ Injury Prevention Program in the 
Collegiate Male Soccer Player. The American Journal of Sports Medicine, 43(11), 2628-37. DoI: https://doi.org/10.1177/0363546515602009

Steffen, K., Bakka, H. M., Myklebust, G., y Bahr, R. (2008). Performance aspects of an injury prevention program: A ten-week intervention in adolescent female football players. Scandinavian Journal of Medicine \& Science in Sports, 18(5), 596-604. Dor: https://doi.org/10.1111/j.1600-0838.2007.00708.x 
